\title{
Neuroimaging's Role in Neuropsychology: Introduction to the Special Issue of Neuropsychology Review on Neuroimaging in Neuropsychology
}

\author{
Edith V. Sullivan ${ }^{1}$ Erin D. Bigler ${ }^{2,3,4,5}$
}

Received: 31 July 2015 / Accepted: 3 August 2015 / Published online: 15 August 2015

(C) Springer Science+Business Media New York 2015

Neuroimaging in the service of neuropsychology is the theme of this special issue of Neuropsychology Review. The field of neuropsychology, as the name implies, examines the neural bases of behavior and cognition with clinical neuropsychology focused exclusively on humans. Traditionally, in neuropsychology it has been sufficient to define the "neuro" segment of neuropsychology as anything neurological — such as postmortem descriptions of lesion location related to antemortem descriptions of behavior and cognition and comparisons of performance by patients with known neurological conditions with performance by healthy controls of similar age and sex. Contrast of groups with presumed neurological abnormalities of a particular lobe or hemisphere together with examination of the individuals with cognitive or other behavioral measures has been a core method for the field. How the field defines the "neuro" component of neuropsychology, however, is evolving and will likely mark its viability as a discipline.

1973 is a landmark date for neuropsychology and neuroimaging. By 1973 understanding hemispheric specialization and cerebral dominance had become markers for how

Edith V. Sullivan

edie@stanford.edu

Erin D. Bigler

erin_bigler@byu.edu

1 Department of Psychiatry \& Behavioral Sciences, Stanford University School of Medicine, Stanford, CA, USA

2 Department of Psychology, Brigham Young University, Provo, UT, USA

3 Neuroscience Center, Brigham Young University, Provo, UT, USA

4 Department of Psychiatry, University of Utah, Salt Lake City, UT, USA

5 Department of Psychology and Neuroscience Center, Brigham Young University, 1001 SWKT, Provo, UT 84602, USA behavioral assessments could be indicators of human brain function (Glass et al. 1973; Goldstein and Shelly 1973; Kimura 1973; Luria 1973; McGlone and Davidson 1973; Teng and Sperry 1973; Zangwill 1973). In that same year, computerized transverse axial tomography was introduced (Ambrose and Hounsfield 1973; Hounsfield 1973). Testament to the innovation characterizing this era was the awarding of Nobel prizes in Physiology or Medicine to Allan Cormack and Godfrey Hounsfield in 1979 "for the development of computed assisted tomography" and to Roger Sperry in 1981 "for his discoveries concerning the functional specialization of the cerebral hemispheres." With the advent of CT and the further work of Hounsfield, modern neuroimaging began, marking a scientific revolution in the neurosciences (Kuhn 1962).

1973 is also a pivotal time in the establishment of the first international society of neuropsychology — open to members through application rather than by invitation - that became what is now the International Neuropsychological Society (INS, see Rourke and Murji 2000). Despite the historical significance of this date, if one searches the National Library of Medicine with the term 'neuropsychology' for the year of 1973, just three publications are found. Likewise, if computerized tomography is searched, only two papers emerge - both by Hounsfield. It would be several more years before the first neuropsychological studies began to define groups by CT imaging. By the early 1980s, CT imaging had become well established, with magnetic resonance imaging (MRI), a safer technology free of ionizing radiation, rapidly coming online. During this time, neuropsychology used imaging, as it had done with other neurological information, to define groups by lesion type, location, size, and even physiological characteristics.

As reviewed by Benton (1994, 2003) and Benton and Sivan (2007), by the 1980s clinical neuropsychology was well 
established and with numerous tests developed to assess a complex array of cognitive, sensory, and motor functions and behaviors (see Lezak 1983). The majority of clinical neuropsychological tests that had their origin in this early era have endured as the mainstay of traditional neuropsychological assessment now in 2015. While these traditional neuropsychological measures may be updated with improved norms, by and large they retain their original test format, theory of brain function, and test items or ones similar to the original.

During this period, neuroimaging acquisition approaches and methods of data analysis multiplied exponentially. From the nascency of neuropsychology and modern neuroimaging in the 1970s, in 2014 there were over 1200 published articles with 'neuropsychology' as the search word, but there were more than 12,000 articles on contemporary 'neuroimaging.' Nuclear medicine used CT technology to develop positron emission tomography (PET) and single photon emission computed tomography (SPECT). MRI started with imaging brain parenchyma but rapidly methods were developed for vascular and functional neuroimaging (fMRI). Improved MRI techniques for identifying various types of pathologies emerged along with methods for assessing metabolic aspects of brain tissue inferred from MR spectroscopy (MRS). Developments in diffusion MRI lead to the development of diffusion tensor imaging (DTI) and techniques that permit the study of major pathways of the brain. Not reviewed in this special issue, there have also been major advances in the allied field of physiology where some advances in electrophysiology and magnetoencephalography parallel those of neuroimaging and can be fully integrated with neuroimaging.

Advances in structural and functional neuroimaging science supplanted earlier, invasive or Xray-based technology, and further technological advances have enabled specific imaging sequences to become standards in clinical diagnosis. For example, the MRI sequence referred to as the fluid attenuate inversion recovery (FLAIR) has proved fundamental in assessing patients with demyelinating disorders. Neuroimaging technology built on the earliest findings, and so if Hounsfield were alive today, he would surely recognize the foundations of his work but likely be surprised by how the neuroimaging field burgeoned and changed. By contrast, Sperry (and other early contemporaries of neuropsychology) would recognize most of the behavioral and cognitive measures to be similar and basically unchanged to what was present in 1973.

So in the 21st Century, how should the 'neuro' in neuropsychology be defined (cf., Sullivan 2009)? In our opinion, neuroimaging will complement the 'psychology' component of neuropsychology, which relies on measurement of observable behavioral responses. Neuroimaging will also enhance the nosology of neuropsychological disorders that has guided much of its research and conceptualization of the neural underpinnings of behavior. Current nosology has generated global categories such as "memory," "executive function," and "visuospatial ability." These conceptualizations have served neuropsychology well in the development of understanding the behavioral side of cognitive domains and will be substantially bolstered with neuroimaging-based understanding of the neural components of these cognitive domains, thereby providing insight into neural mechanisms of selective behaviors. Indeed, we predict that in 21 st Century neuropsychology it will be insufficient to say a patient has, for example, an executive function deficit, when neuroimaging has the potential to identify damaged or dysfunctional brain regions, neural systems, and pathways underlying the observed behavioral impairment. Identification of neural mechanisms of disruption can reveal targets for repair.

Ever observant, responsible in scholarship, and forward thinking Arthur Benton, a 20th Century father of neuropsychology, wrote,

The neurosciences, both basic and applied, advance at a pace so precipitous as to be almost disorienting. We are proud of today's CT, MRI and PET which have been serving us so well in the laboratory and the clinic. But there can be little doubt that these techniques, which not long ago were seen as revolutionary developments (which they were) will be displaced by equally revolutionary new instrumentation and procedures. Twentyfive years from now, neuroscientists will be addressing basic questions of the nature of neural activity, the functional organization of the units of the nervous system and the age-old 'mind-body' problem in far greater depth and in a much more effective way than is now possible.

"Soon enough we will be 'history" (Benton 2003, pp. 30-31).

In this issue of Neuropsychology Review, leading imaging scientists from the integrated field of neuropsychology and cognitive neuroscience provide frameworks for how advanced neuroimaging methods can accelerate discovery and become a routine part of neuropsychology.

The paper by Bigler presents a brief history and fundamentals of structural MRI and thoughts about testing brain structure-function associations and dissociations. Mueller, Lim, Hemmy, and Camchong review concepts underlying MR DTI, used principally to assess microstructural integrity of white matter tracts, and provide an overview of applications in normal aging and neuropsychiatric disorders. Daugherty and Raz introduce MR susceptibility-weighted imaging, which enables detection, localization, and quantification of iron deposition in the brain, which accrues with aging, is over-represented in selective diseases, and has relations with cognitive and motor impairment. Chen and Glover provide the essentials of fMRI and relate scopes and limits of this popular 
adjunct of neuropsychology. Ende reviews MRS, which is a noninvasive method for detecting major brain metabolites, typically on the proton spectrum; her review focuses on GABA and glutamate, which are essential biochemicals with a small in vivo signal, making them highly challenging to quantify. Segobin, La Joie, Ritz, Beaunieux, Desgranges, Chelelat, Pitel, and Eustache review PET, one of the earliest functional neuroimaging methods used in neuropsychology and still a useful tool in detecting and tracking diseases of the brain. The final paper by Nichols and Pohl describes biomedical informatics as a burgeoning tool employing a constellation of methods used for data storage, retrieval, and analysis of large or small data sets and essential for data distribution, which is a growing requirement of granting agencies and peerreview journals.

These reviews present a sampling of 21 st century imaging approaches and informatics considerations for identifying the "neuro" in neuropsychology.

Acknowledgments Dr. Bigler used no grant funds in writing this editorial; he co-directs the Neuropsychological Assessment and Research Laboratory at Brigham Young University, which provides forensic consultation.

Dr. Sullivan acknowledges NIAAA funding during the writing of this editorial (K05 AA017168).

\section{References}

Ambrose, J., \& Hounsfield, G. (1973). Computerized transverse axial tomography. The British Journal of Radiology, 46(542), 148-149.

Benton, A. (1994). Four neuropsychologists. [Biography Historical Article]. Neuropsychology Review, 4(1), 31-44.
Benton, A. (2003). Recollections of a part-time amateur neurohistorian. [Autobiography Biography Historical Article Portraits]. Journal of the History of the Neurosciences, 12(1), 25-33. doi:10.1076/jhin.12. 1.25.13783.

Benton, A. L., \& Sivan, A. B. (2007). Clinical neuropsychology: a brief history. [Historical Article]. Disease-a-Month: DM, 53(3), 142-147. doi:10.1016/j.disamonth.2007.04.003.

Glass, A. V., Gazzaniga, M. S., \& Premack, D. (1973). Artificial language training in global aphasics. Neuropsychologia, 11(1), 95-103.

Goldstein, G., \& Shelly, C. H. (1973). Univariate vs. multivariate analysis in neuropsychological test assessment of lateralized brain damage. Cortex, 9(2), 204-216.

Hounsfield, G. N. (1973). Computerized transverse axial scanning (tomography). 1. Description of system. The British Journal of Radiology, 46(552), 1016-1022. doi:10.1259/0007-1285-46-5521016.

Kimura, D. (1973). Manual activity during speaking. II. Left-handers. Neuropsychologia, 11(1), 51-55.

Kuhn, T. S. (1962). The structure of scientific revolutions. Chicago: University of Chicago Press.

Lezak, M. D. (1983). Neuropsychological assessment. New York: Oxford University Press.

Luria, A. R. (1973). Neuropsychological studies in the USSR. A review. I. [Review]. Proceedings of the National Academy of Sciences of the United States of America, 70(3), 959-964.

McGlone, J., \& Davidson, W. (1973). The relation between cerebral speech laterality and spatial ability with special reference to sex and hand preference. Neuropsychologia, 11(1), 105-113.

Rourke, B. P., \& Murji, S. (2000). A history of the International Neuropsychological Society: the early years (1965-1985). [Historical Article]. Journal of the International Neuropsychological Society: JINS, 6(4), 491-509.

Sullivan, E. V. (2009). Neuropsychology review: a resource for the clinical, experimental, and translational neuropsychologists. Neuropsychology Review, 19, 1-3.

Teng, E. L., \& Sperry, R. W. (1973). Interhemispheric interaction during simultaneous bilateral presentation of letters or digits in commissurotomized patients. Neuropsychologia, 11(2), 131-140.

Zangwill, O. L. (1973). R. C. Oldfield's contribution to neuropsychology. [Biography Historical Article]. Neuropsychologia, 11(4), 373-376. 